\title{
Antimicrobial susceptibility of Mycoplasma genitalium isolates from Pretoria, South Africa in 2012 and 2016
}

\author{
Marie C Le Roux ${ }^{a *}$, Maanda Mafunise ${ }^{a}$, Barbara E de Villiers ${ }^{a}$ and Ramalau MM Ditsele ${ }^{a b}$ \\ ${ }^{a}$ Department of Microbiological Pathology, Sefako Makgatho Health Sciences University, Ga-Rankuwa, South Africa \\ ${ }^{b}$ NHLS Dr George Mukhari Academic Hospital, Ga-Rankuwa, South Africa \\ *Corresponding author, email: marcelle.leroux@smu.ac.za
}

Background: Mycoplasma genitalium is a sexually transmitted pathogen associated with non-gonococcal urethritis and cervicitis. Azithromycin regimens have been considered first-line treatment for $M$. genitalium infections, with fluoroquinolone regimens effective as second-line treatments. However, the proportion of $M$. genitalium harbouring macrolide or fluoroquinolone resistance-associated mutations has been increasing worldwide. This study was done to compare the genotypic macrolide and fluoroquinolone resistance of $M$. genitalium strains obtained from women attending a termination of pregnancy clinic five years apart.

Methods: M. genitalium was detected by PCR in vaginal swab samples from 100 and 104 termination of pregnancy attendees at a tertiary hospital in Pretoria, South Africa during 2012 and 2016 respectively. Genes associated with macrolide and fluoroquinolone resistance in the $M$. genitalium isolates were sequenced and analysed.

Results: The prevalence of M. genitalium was $6.0 \%$ (6/100) in 2012 and $7.7 \%(8 / 104)$ in 2016. No resistance-associated mutations were seen in the 2012 isolates. Among the 2016 M. genitalium isolates, two (25\%) harboured a macrolide-associated resistance mutation and one (12.5\%) a fluoroquinolone resistance-associated mutation in the parC gene.

Conclusions: There is an increase in macrolide and fluoroquinolone resistance among local M. genitalium strains. This highlights the need for improved surveillance.

Keywords: fluoroquinolone resistance, macrolide resistance, Mycoplasma genitalium, South Africa, termination of pregnancy clinic

\section{Background \\ Mycoplasma genitalium is a sexually transmitted pathogen associated with non-gonococcal urethritis, cervicitis, and related upper genital tract conditions such as pelvic inflammatory disease and infertility. ${ }^{1,2}$ M. genitalium is the smallest self- replicating prokaryote, but despite its small size, it is capable of causing disease, evades host immune responses through antigenic variability, and develops resistance to antimicrobial agents. $^{3}$}

Routine screening for $M$. genitalium is performed in only a few countries. As the organism cannot readily be cultured on standard laboratory media, detection relies on nucleic acid amplification tests. Although there are a few commercial tests available, there are still no assays approved by the FDA for routine diagnostic testing. ${ }^{4}$ In most cases of sexually acquired urethritis and cervicitis, tests are only performed for Neisseria gonorrhoeae and Chlamydia trachomatis.

The treatment of M. genitalium infection, either individually or as part of syndromic management, differs globally. Although no genetic determinant such as tet $M$ has been found in this mycoplasma, tetracyclines are not useful for in vivo eradication of $M$. genitalium, with reported cure rates of around $30 \% .{ }^{5,6}$ The efficacy of doxycycline, used extensively to treat non-gonococcal urethritis and cervicitis in the past, is relatively poor for $M$. genitalium infection. Azithromycin has been the preferred treatment for several years in many countries.

As with other bacterial sexually transmitted infections (STIs), such as gonorrhoea, antimicrobial resistance poses a threat to the effective treatment of $M$. genitalium. Treatment failure due to the rapid emergence of macrolide resistance is now very common and widespread. ${ }^{7}$ Where studies have shown that older fluoroquinolones such as ciprofloxacin and levofloxacin are less active against $M$. genitalium, moxifloxacin has been demonstrated to be effective in patients with macrolide-resistant strains of $M$. genitalium. ${ }^{8}$ However, fluoroquinolone resistance, including resistance to moxifloxacin, has also been described. ${ }^{8}$

In South Africa, the recommended management of urogenital infections is syndromic. The recommended seven-day course of doxycycline for genital discharge syndromes (i.e. male urethritis and vaginal discharge) was changed in 2015 to include singledose oral azithromycin only, with doxycycline no longer in the guidelines for use other than for penicillin allergy in syphilis and balanitis.

Mutations in specific genes may be associated with resistance to antimicrobial drugs. Resistance to macrolides was shown to be due to mutations occurring in region $\mathrm{V}$ of the $23 \mathrm{~S}$ rRNA gene, ${ }^{9}$ and fluoroquinolone resistance due to mutations in the gyrA and parC genes ${ }^{10}$ of $M$. genitalium.

In our study, we compared the macrolide and fluoroquinolone resistance profiles of $M$. genitalium strains obtained from woman attending a termination of pregnancy clinic in 2012 with those attending in 2016.

\section{Materials and methods Patient samples and processing}

Patients visiting the termination of pregnancy clinic (TOP) at the Dr George Mukhari Academic Hospital (DGMAH) in Pretoria, 
South Africa were recruited for the study. Consecutive consenting women were enrolled both in 2012 (August) and in 2016 (September). Both groups of patients had not received any antibiotic therapy in the preceding month and gave verbal consent for participation. After a general examination, vaginal swab specimens were collected by a clinician and delivered to the laboratory within 24 hours. DNA was extracted from the swabs using the ZR Genomic DNA ${ }^{\mathrm{TM}}$-Tissue MiniPrep kit (Zymo Research Corp, Irvine, CA, USA) according to the manufacturer's instructions for biological liquids and cell suspensions.

\section{Molecular assays}

DNA from the ATCC M. genitalium G37 strain (33530D) was included in all PCR assays as positive control, and PCR grade water (Bioline) was used as negative control. The presence of $M$. genitalium was detected using conventional PCR as described previously. ${ }^{11}$ The primers targeted a $281 \mathrm{bp}$ region of the $140 \mathrm{kDa}$ adhesion gene $(\mathrm{MgPa})$ of $\mathrm{M}$. genitalium.

Antimicrobial resistance genes were analysed in all M. genitalium positive isolates. Potential macrolide resistance was detected by amplifying and sequencing a unique $147 \mathrm{bp}$ region of the $\mathrm{V}$ region of the $23 \mathrm{~S}$ rRNA of $M$. genitalium. ${ }^{9}$ BLAST technology was used to compare sequences with the $M$. genitalium $\mathrm{G} 37$ complete genome [L43967.2]. Sequences of strains with known mutations were also used in the analysis: LA141 [HF572938.1] (A2058G); LA088 [HF572933.1] (A2059G) and LA202 [HF572946.1] (A2059C). GyrA and parC genes were amplified and sequenced to detect fluoroquinolone-associated resistance mutations (M95I and D99 N in gyrA; S83I, S83R, and D87 N in parC). Primers targeting a $230 \mathrm{bp}$ region of the $g y r A$ gene and a $220 \mathrm{bp}$ region of the parC gene were used. ${ }^{10}$ BLAST technology was used to compare sequences with DNA gyrase subunit A [M. genitalium G37; ID: L43967.2]; the whole genome [M. genitalium MG6320; ID:CP003772.1]; and topoisomerase IV, A subunit [M. genitalium strain LA107; ID: HF947096.1].

Descriptive statistical analysis was performed using SPSS ${ }^{\oplus}$ version 16 (SPSS Inc, Chicago, IL, USA) to compare results obtained in 2012 and in 2016. Sequences obtained were edited using Chromas Lite and BioEdit. Sequences were aligned with MAFFT.

\section{Results}

One hundred samples were collected in September 2012 and 104 in October 2016. The median age of the women was 23 years (range: 18-42 years); with the mean ages of the women 24 and 23 years in 2012 and 2016 respectively. M. genitalium was detected in six of the 100 specimens (6.0\%) in 2012 and in eight of 104 (7.7\%) specimens in 2016.

The $V$ region of the $23 \mathrm{~S}$ rRNA of $M$. genitalium was amplified and sequenced for 13 isolates (five in 2012 and eight in 2016). None of the 2012 isolates harboured macrolide resistance-associated mutations. Among the 2016 isolates, two isolates had the A2059G mutation. This is one of the three different mutations at positions 2058 and 2059 (E. coli numbering) in region $V$ of the $23 \mathrm{~S}$ rRNA gene that have been associated with the macrolideresistance phenotype.

Twelve gyrA and parC positive amplicons were sequenced (five in 2012 and seven in 2016). Neither the 2012 nor 2016 strains harboured mutations in the gyrA gene associated with fluoroquinolone resistance as reported previously.12
Table 1. Genotypic resistance of M. genitalium isolates

\begin{tabular}{|c|c|c|c|c|}
\hline \multirow[t]{2}{*}{ Year } & \multirow[t]{2}{*}{ Patient ID } & \multirow[t]{2}{*}{$\begin{array}{l}23 S \text { rRNA } \\
\text { mutation }\end{array}$} & \multicolumn{2}{|c|}{$\begin{array}{l}\text { Mutant fluoroquinolone } \\
\text { QRDR amino acid change }\end{array}$} \\
\hline & & & GyrA & ParC \\
\hline \multirow[t]{6}{*}{2012} & M13 & WT & WT & WT \\
\hline & M35 & WT & ND & ND \\
\hline & M63 & WT & WT & $\begin{array}{l}\text { C234T (no } \\
\text { change) }\end{array}$ \\
\hline & M72 & WT & WT & WT \\
\hline & M78 & ND & WT & WT \\
\hline & M98 & WT & WT & WT \\
\hline \multirow[t]{9}{*}{2016} & $\mathrm{~T} 25$ & A2059G & WT & WT \\
\hline & \multirow[t]{2}{*}{ T30 } & \multirow[t]{2}{*}{ A2059G } & \multirow[t]{2}{*}{ WT } & $\begin{array}{l}\text { C234T (no } \\
\text { change) }\end{array}$ \\
\hline & & & & $\begin{array}{c}\mathrm{G} 248 \mathrm{~T}(\mathrm{Ser} \rightarrow \| \mathrm{le} \\
[83])\end{array}$ \\
\hline & T37 & WT & ND & ND \\
\hline & T63 & WT & WT & $\begin{array}{l}\text { C234T (no } \\
\text { change) }\end{array}$ \\
\hline & T71 & WT & WT & WT \\
\hline & T75 & WT & WT & WT \\
\hline & T76 & WT & WT & $\begin{array}{l}\text { C234T (no } \\
\text { change) }\end{array}$ \\
\hline & T101 & WT & WT & WT \\
\hline
\end{tabular}

Note: WT: wild type; ND: not done.

None of the 2012 isolates harboured the parC alterations associated with fluoroquinolone resistance, while one strain from 2016 had the G248T resistance-associated mutation (Ser $\rightarrow$ lle 83) which has been previously described for $M$. genitalium. ${ }^{12}$ One strain in 2012 and three strains in 2016 shared the same silent mutation (C234T) in the parC gene. Results are summarised in Table 1.

\section{Discussion}

The prevalence of $M$. genitalium among women attending for TOP at DGMAH did not change much over the five years between the studies $(6.0 \%$ vs. $7.7 \%)$. This was similar to the prevalence of vaginal M. genitalium (8.7\% [52/601]) in women visiting primary health care clinics across the Mopani District of Limpopo province in $2015 .^{14}$ The $M$. genitalium prevalence patterns tend to differ between studies, with rates ranging from $0 \%$ to as high as $47.5 \%$ in different population samples. ${ }^{15,16}$ Data collected from various studies concerning the prevalence of $M$. genitalium infections are somewhat inconsistent and contradictory and this is mainly due to the heterogeneity in populations studied, sampling methods used, underlying risk factors for infection and clinical presentation (symptomatic or asymptomatic). ${ }^{15}$ In a study at a TOP clinic in Denmark, $M$. genitalium was detected in less than $1 \%$ of the 102 participants ${ }^{17}$ while the organism was detected in $22.4 \%$ of women recruited from an urban medical centre in Cincinnati, USA. ${ }^{18}$ As our study was not done among patients with vaginal discharge syndrome but rather among women seeking termination of pregnancy, the prevalence and probably bacterial loads of $M$. genitalium were relatively low. A South African study conducted on women with vaginal discharge syndrome from Cape Town and Johannesburg reported a significantly higher M. genitalium prevalence among participants from Johannesburg than those from Cape Town (11.2\% vs. $2.1 \%$; $p=0.0066){ }^{19}$ 
Like all mycoplasmas, $M$. genitalium has natural resistance against all beta-lactam agents and other antibiotics targeting peptidoglycan assembly since they lack a cell wall. Treatment options are tetracyclines, macrolides, and fluoroquinolones. Although doxycycline has been used to treat nongonococcal urethritis and cervicitis for many years, it has been shown to have poor efficacy against $M$. genitalium and isolates with reduced susceptibility have been reported. ${ }^{5}$ The macrolide azithromycin is now preferred for the treatment of nongonococcal urethritis and related clinical syndromes. However, after the first report in 2006 of azithromycin resistance observed in M. genitalium isolates among Australian patients, ${ }^{20}$ the efficacy of the drug in treating M. genitalium infections has decreased worldwide. ${ }^{21}$

Resistance to macrolides is believed to be associated with point mutations occurring in region 5 (referred to as ' $V$ region') of the $23 \mathrm{~S}$ rRNA. Three mutations at positions 2058 and 2059 (E. coli numbering) have been reported. ${ }^{9}$ In our study, none of the 2012 isolates harboured macrolide resistance-associated mutations, while two of the 2016 isolates (25.0\%) had the A2059G mutation, which is associated with macrolide resistance. This was also the most commonly seen resistance-associated mutation reported from Australia. ${ }^{12}$ Ours is the second report of macrolide resistance in South Africa. Macrolide resistance-associated mutations were previously reported in 4/41 (9.8\%) in M. genitalium-positive specimens collected from women attending primary health care clinics in the Limpopo province of rural South Africa. ${ }^{14}$ Contrary to our study, all their mutated specimens harboured the A2058G mutation.

The presence of macrolide resistance is of concern, as the treatment guidelines issued by the South African Department of Health were changed in 2015 , when doxycycline was replaced by a single dose of azithromycin for the treatment of male urethritis and vaginal discharge syndromes. Although the prevalence of $M$. genitalium among the patients in our study was similar over the five-year period, macrolide resistance increased. This trend is observed in many countries, with the proportion of cases of $M$. genitalium with these mutations increasing in recent years. Data from Sweden showed the proportion of infections with a mutation increasing from $0 \%$ in 2006 to $14 \%$ in 2010 , and $21 \%$ in 2011.22 In Japan an increase from 0\% in 2011 and 2012 to 29\% in 2013 was reported ${ }^{23}$ and in Australia an increase from about 20\% between 2007 and 2009 to 36\% between 2012 and 2013 was shown..$^{24}$ It is believed that the continued use of azithromycin $1 \mathrm{~g}$ therapy is driving the increase in macrolide antimicrobial resistance as a result of frequent selection of resistant genotypes. ${ }^{21}$ This has led some investigators to use an extended dose of azithromycin, in the belief that it may be less likely to induce resistance than a single $1 \mathrm{~g}$ dose. ${ }^{8,25}$

Following treatment failure by macrolides, patients with macrolide-resistant strains of $M$. genitalium are usually treated with fluoroquinolones, particularly moxifloxacin. ${ }^{7}$ This fourthgeneration quinolone drug is bactericidal and generally well tolerated. In our study, none of the strains harboured mutations in the gyrA gene. One M. genitalium isolate from 2016 harboured a fluoroquinolone resistance-associated mutation in the parC gene, which is the first reported in South Africa. This G248T mutation (Ser $\rightarrow$ lle 80) is among those that are known to be associated with fluoroquinolone resistance in $M$. genitalium and other closely related organisms. ${ }^{13,26}$ This mutation was common in M. genitalium isolates from patients attending sexual health clinics in Sydney, Australia, ${ }^{12}$ as well as among Japanese men with male urethritis syndrome ${ }^{23}$ and patients from France. ${ }^{27}$ Although we did not perform susceptibility testing to confirm phenotypic resistance, based on previous studies it is likely that the mutation observed in our isolate will contribute to moxifloxacin resistance. ${ }^{13}$

One strain in 2012 and three strains in 2016 shared the same silent mutation (C234T) in the parC gene of M. genitalium. This mutation was also commonly found among isolates in Japan, but the significance of this mutation is unknown. ${ }^{13,26}$

Resistance of M. genitalium to macrolides and fluoroquinolones in our study showed that in South Africa a similar trend is observed as reported for the Asia-Pacific region where escalating resistance to azithromycin and moxifloxacin for M. genitalium has been seen. Analysing 140 infections, combined macrolide/fluoroquinoloneresistant mutations were found in $8.6 \%$ of specimens, for which recommended therapies would be ineffective..$^{28}$

Limitations of this study include the small numbers of $M$. genitalium isolates obtained during this period that could be tested, and the absence of a clinical history from participants, especially on the treatment for genital discharge syndrome in the preceding period. More surveillance data over time are needed from both symptomatic and asymptomatic patients to describe trends in macrolide and fluoroquinolone resistance accurately.

In conclusion, macrolide-resistant $M$. genitalium was present in this population and fluoroquinolone resistance was seen for the first time. As the prevalence of M. genitalium and the associated number of antimicrobial resistance markers was low, this study still does not justify changing the South African treatment guidelines for symptomatic patients with M. genitalium. However, as data for M. genitalium are not widely available in South Africa this and future studies should pave the way towards improved antimicrobial surveillance in M. genitalium.

Acknowledgement - This study was presented in part at the STI \& HIV World Congress, Rio de Janeiro, Brazil, in July 2017.

Funding - The study was funded by VLIR.

Ethical approval - Ethical approval for the study was obtained from the Medunsa Research and Ethics Committee (MREC) of the University of Limpopo (Medunsa Campus): MCREC/P/85/2007:PG and SMUREC/P/138/2015:PG.

Disclosure statement - No potential conflict of interest was reported by the authors.

\section{References}

1. Taylor-Robinson D, Jensen JS. Mycoplasma genitalium: from chrysalis to multicolored butterfly. Clin Microbiol Rev. 2011;24(3):498-514. https://doi.org/10.1128/CMR.00006-11

2. Manhart LE. Mycoplasma genitalium: an emergent sexually transmitted disease? Infect Dis Clin North Am. 2013;27(4):779-92. https://doi.org/10.1016/j.idc.2013.08.003

3. Razin S, Yogev D, Naot Y. Molecular biology and pathogenicity of mycoplasmas. Microbiol Mol Biol Rev. 1998;62(4):1094-156.

4. Le Roy C, Pereyre S, Bebear C. Evaluation of two commercial real-time PCR assays for detection of Mycoplasma genitalium in urogenital specimens. J Clin Microbiol. 2014;52(3):971-3. https://doi. org/10.1128/JCM.02567-13

5. Hamasuna R, Jensen JS, Osada Y. Antimicrobial susceptibilities of Mycoplasma genitalium strains examined by broth dilution and quantitative PCR. Antimicrob Agents Chemother. 2009;53(11):4938-9. https://doi.org/10.1128/AAC.00724-09 
6. Falk L, Fredlund $\mathrm{H}$, Jensen JS. Tetracycline treatment does not eradicate Mycoplasma genitalium. Sex Transm Infect. 2003;79(4):318-9. https://doi.org/10.1136/sti.79.4.318

7. Weinstein S, Stiles B. Recent perspectives in the diagnosis and evidence-based treatment of Mycoplasma genitalium. Expert Rev Anti Infect Ther. 2012;10:487-99. https://doi.org/10.1586/eri.12.20

8. Jernberg E, Moghaddam A, Moi H. Azithromycin and moxifloxacin for microbiological cure of Mycoplasma genitalium infection: an open study. Int J STD AIDS. 2008;19:676-9. https://doi.org/10.1258/ijsa.2008.008038

9. Jensen JS, Bradshaw CS, Tabrizi SN, et al. Azithromycin treatment failure in Mycoplasma genitalium - Positive patients with nongonococcal urethritis is associated with induced macrolide resistance. Clin Infect Dis. 2008;47(12):1546-53. https://doi.org/10.1086/596321

10. Deguchi T, Maeda S, Tamaki M, et al. Analysis of the gyrA and parC genes of Mycoplasma genitalium detected in first-pass urine of men with non-gonococcal urethritis before and after fluoroquinolone treatment. J Antimicrob Chemother. 2001;48(5):742-4. https://doi.org/10.1093/jac/48.5.742

11. Jensen JS, Uldum SA, Søndergård-Andersen J, Vuust J, Lind K. Polymerase chain reaction for detection of Mycoplasma genitalium in clinical samples. J Clin Microbiol. 1991;29(1):46-50.

12. Tagg KA, Jeoffreys NJ, Couldwell DL, et al. Fluoroquinolone and macrolide resistance-associated mutations in Mycoplasma genitalium. J Clin Microbiol. 2013;51(7):2245-9. https://doi.org/10.1128/JCM.00495-13

13. Shimada Y, Deguchi T, Nakane K, et al. Emergence of clinical strains of Mycoplasma genitalium harbouring alterations in ParC associated with fluoroquinolone resistance. Int J Antimicrob Agents. 2010;36(3):255-8. https://doi.org/10.1016/j.ijantimicag.2010.05.011

14. Hay B, DubbinkJH, Ouburg S, etal.Prevalence and macrolide resistance of Mycoplasma genitalium in South African women. Sex Transm Dis. 2015;42(3):140-2. https://doi.org/10.1097/OLQ.0000000000000246

15. Daley GM, Russell DB, Tabrizi SN, et al. Mycoplasma genitalium: a review. Int J STD \& AIDS. 2014;25(7):475-87. https://doi. org/10.1177/0956462413515196

16. McGowin CL, Anderson-Smits C. Mycoplasma genitalium: An emerging cause of sexually transmitted disease in women. PLoS Pathog. 2011;7(5):e1001324. https://doi.org/10.1371/journal. ppat.1001324

17. Baczynska A, Hvid M, Lamy P, et al. Prevalence of Mycoplasma genitalium, Mycoplasma hominis and Chlamydia trachomatis among danish patients requesting abortion. Syst Biol Reprod Med. 2008;54(3):127-34. https://doi.org/10.1080/19396360802042838
18. Huppert JS, Mortensen JE, Reed JL, et al. Mycoplasma genitalium detected by transcription-mediated amplification is associated with Chlamydia trachomatis in adolescent women. Sex Transm Dis 2008;35(3):250-4. https://doi.org/10.1097/OLQ.0b013e31815abac6

19. Mhlongo S, Magooa $P$, Müller EE, et al. Etiology and STI/HIV coinfections among patients with urethral and vaginal discharge syndromes in South Africa. Sex Transm Dis. 2010;37(9):566-70. https://doi.org/10.1097/OLQ.0b013e3181d877b7

20. Bradshaw CS, Jensen JS, Tabrizi SN, et al. Azithromycin failure in Mycoplasma genitalium urethritis. Emerg Infect Dis. 2006;12(7):114952. https://doi.org/10.3201/eid1207.051558

21. Lau A, Bradshaw CS, Lewis D, et al. The efficacy of azithromycin for the treatment of genital Mycoplasma genitalium: a systematic review and meta-analysis. Clin Infect Dis. 2015;61(9):1389-99. https://doi.org/10.1093/cid/civ644

22. Anagrius C, Loré B, Jensen JS. Treatment of Mycoplasma genitalium. Observations from a Swedish STD clinic. PLoS ONE. 2013;8:e61481. https://doi.org/10.1371/journal.pone.0061481

23. Kikuchi $M$, Ito $S$, Yasuda $M$, et al. Remarkable increase in fluoroquinolone-resistant Mycoplasma genitalium in Japan. J Antimicrob Chemother. 2014;69(9):2376-82. https://doi.org/10.1093/ jac/dku164

24. Bissessor M, Tabrizi SN, Twin J, et al. Macrolide resistance and azithromycin failure in a Mycoplasma genitalium-infected cohort, and response of azithromycin failures to alternative antibiotic regimens. Clin Infect Dis. 2015;60:1228-36. https://doi.org/10.1093/cid/ciu1162

25. Terada M, Ohki Emiko, Yamagishi Yuka, et al. Antimicrobial efficacies of several antibiotics against uterine cervicitis caused by Mycoplasma genitalium. J Inf Chemother. 2012;18(3):313-7. https://doi.org/10.1007/s10156-011-0329-8

26. Gruson D, Pereyre S, Renaudin $\mathrm{H}$, et al. In Vitro development of resistance to six and four fluoroquinolones in Mycoplasma pneumoniae and Mycoplasma hominis, respectively. Antimicrob Agents Chemother. 2005;49(3):1190-3. https://doi.org/10.1128/ AAC.49.3.1190-1193.2005

27. Le Roy C, Hénin N, Pereyre S, et al. Fluoroquinolone-resistant Mycoplasma genitalium. Emerg Infect Dis. 2016;22(9):1677-9. https://doi.org/10.3201/eid2209.160446

28. Murray GL, Bradshaw CS, Bissessor M, et al. Increasing macrolide and fluoroquinolone resistance in Mycoplasma genitalium. Emerg Infect Dis. 2017;23(5):809-12. https://doi.org/10.3201/eid2305.161745

Received: 16-05-2017 Accepted: 10-10-2017 